\title{
Membership enforcement as a driver of the evolution of language
}

\author{
Daniil Ryabko \\ INRIA, France (on leave from) \\ Fishlife Research \\ daniil@ryabko.net
}

\author{
Alvaro Mbeju Moreno \\ Fishlife Research \\ Universidad de Asuncion \\ alvaro.mbeju.moreno@gmail.com
}

\begin{abstract}
A novel hypothesis concerning language evolution is advanced. It posits that languages have evolved as a means of binding individuals to a group, as well as for defining those groups. This means that language evolution has to be considered on the level of groups and not only on the level of individuals. This hypothesis helps to explain the huge diversity of human languages, as well as their complexity. Perhaps more importantly, it explains why adults lose the ability to learn languages with the ease that children possess.
\end{abstract}

The evolution of language is a subject that has fascinated researchers right since the Darwin's foundational work. Some of the most intriguing aspects of human languages have been their diversity and complexity. So far, most of the research had explicitly or implicitly assumed that the main functions of the language are communication and (internal) representation of thought (I-language, Chomsky, 2015). Here we consider a very different view, suggesting that the main driver of the evolution of language has been the need for social identity. More specifically, language evolved in such a way as to make it difficult to learn as an adult. Consider the fact that children have the ability to learn a language perfectly and the adults do not, in the sense that it is extremely difficult or often impossible for an adult to learn to speak a language as well as someone who learned it as a child. The cornerstone question that we pose is the following:

Can the loss of language-learning ability in adults be adaptive?

In other words, can the loss of this ability serve some purpose, and if so then is it possible that languages have evolved to fulfill this purpose? Put this way, the question may appear baffling, since it is difficult to imagine what advantage a loss of a cognitive ability may confer to an individual. However, the advantages become clear if this question is considered on the level of 
groups. The loss of language-learning ability defines, for each language, the group of its native speakers, and effectively binds them to this group. Binding individuals to a group allows for the evolution of altruistic behaviour via group-level selection.

Let us consider this latter point in more detail. Group-level (or, more generally, multilevel) selection is used to explain the evolution of altruistic behaviour. Altruistic behaviour is a behaviour that is detrimental to the individual but is beneficial to others which may be only distantly related; non-altruistic behaviour is called selfish. If selection acts on the superindividual level, that is, if groups compete between themselves, then groups which have more altruists get an advantage, even though an altruist is disadvantaged within their group so long as it has at least one selfish member. It is worth noting that the idea of group-level selection as an explanation for the emergence of altruistic behaviour can be traced back to Darwin (Darwin, 1871). Mathematical models of various complexity have been proposed for this phenomenon, demonstrating its viability under certain combinations of the parameters; see (Rand and Nowak, 2013) for a review.

In nature, groups do not necessarily have constant composition: individuals, both altruistic and selfish, may migrate from one group to another. Intuitively, it is clear that migration works against cooperation; specifically, one might think of individuals fleeing a group in the face of danger, e.g. under attack by another group. Mathematical modelling not only confirms this intuition, but shows that even random migration has this effect. One of the simplest models that allows for migration is that by (Traulsen and Nowak, 2006). In this model, the population is divided into groups and individuals reproduce within the groups. Groups may split upon reaching a certain size, and individuals may migrate at random with a certain probability. The authors show that group-level selection favours altruism if $b / c>1+z+n / m$, where $b / c$ is the ratio between the benefit and cost of the altruistic act, $n$ and $m$ denote the maximum group size and the number of groups, and, finally, $z$ is the average number of migrants arising from one group during its lifetime. Thus, at least in this model, migration works directly against altruism: the higher the migration $(z)$ the bigger the benefit of altruism should be related to its cost $(b / c)$ in order for altruism to survive.

Group-level selection in our own species had almost certainly taken place; Tattersall (2016) notes that the spread of Homo sapiens over the planet between $100 \mathrm{kyr}$ and $50 \mathrm{kyr}$ ago occurred far too rapidly to be accounted for by the slow workings of natural selection at the individual level; furthermore, he suggests an explanation based on the invention of langauge (but does not consider its role as a group-binding tool).

If binding individuals to groups is an essential part of group evolution, and in particular of the evolution of altruism, then one should expect there to be more than one means of achieving it. Some examples of the manifestations of this phenomenon can be found in artificial body deformations. 
These have been found across a wide variety of human cultures, and include cranial deformations, foot-binding, scarification, tattooing and so on, which can often be linked to class, status, religion or other forms of social identity (Mackie, 1996; Hoshower et al., 1995; Ludvico and Kurland, 1995). While it is clear that these phenomena are cultural, their widespread presence in only distantly related populations is an evidence of an underlying common genetic adaptation. It is worth nothing that many of the artificial body deformations mentioned are applied to young children, i.e., at the same age that language acquisition starts; others are applied at adolescence, where group membership is being ascertained (e.g., as a right of passage). The presence of these group-binding practices provides an indirect support to the hypothesis advanced in this note.

\section{Discussion}

The fact that language is an important part of social identity is well established. However, its role as a tool to bind individuals to a group and thus in the evolution of altruism so far has not been recognised. Here we hypothesise that this role is the main driver of language evolution. The need for languages to be complex is a direct consequence of this hypothesis. Moreover, for group-level selection to take place, groups needs to reproduce, which entails language diversity. Thus, the proposed hypothesis fits well the empirically observed phenomena (namely, the complexity and diversity of languages) that have remained so far enigmatic form the theory point of view. Moreover, it is supported by theoretical models of group evolution as well as by the evidence of other group-binding practices that permeate human populations around the world.

There are many questions left to be answered. Most importantly, while the proposed hypothesis attempts to explain why the language evolved and what purposes it serves, it remains so far to speculate exactly how this evolutionary process took place. One can envisage two distinct possibilities: either the language evolved on the basis on some pre-existing cognitive abilities that children possess and that they lose growing up; or, the evolutionary process has somehow suppressed some existing cognitive ability; or, which is most likely, it was some combination of the two. If some pre-existing abilities played the main role, we would perhaps be able to find some other cognitive abilities that children have but adults do not. These appear rather difficult to find in humans. However, looking beyond our own species, a related mechanism can be found, namely that of imprinting. It is widespread in the so-called precocial species, i.e. those in which the young are born relatively mature. Specifically, filial imprinting establishes social preference for an object that should typically be the parent, during the first hours or days of life; sexual imprinting works similarly to establish sexual preference (see, 
e.g., Reznikova (2007) for review). Most importantly, these mechanisms are only active during certain sensitive periods of life. Clearly, humans are not precocial species and do not need to imprint the parental image for the same reasons that gees chicks do. However, similar cognitive mechanisms could be present. Sexual imprinting is also interesting to consider from this point of view, as the choice of sexual partner is largely affected by their group membership and social standing. Moreover, if we consider the process of reproduction of groups themselves, and thus of reproduction of languages, we may notice that there is the second period that is sensitive for group identification: the adolescence. Adolescents are susceptible to create their own jargons and accents; understanding how this process is related with and affects sexual partner choice may be key to further understanding the process of language evolution.

Another question that acquires a new perspective from the point of view of the proposed hypothesis is the exclusivity of the capacity for language to our species. For a long time, language has been considered a uniquely human faculty, and, to a large extent, this view persists to this day, see Corballis (2017) for a critical review. From the perspective of the evolution of groups and altruism, our species does appear to be unique, though by no means the most advanced. Humans are perhaps the only species to live in large, highly-structured but at the same time genetically heterogeneous communities. Eusocial species, such as bees, ants and mole rats, are more advanced in the sense that their communities are much more genetically homogeneous. Some of these latter species, in particular bees and a select few species of ants, possess languages that allow for transmission of quantitative information, and, at least in the case of ants, are flexible enough to allow for information compression (review in Reznikova and Ryabko (2011)). Since they are eusocial, the groups in these species are families, i.e., are bound genetically, and so do not need to bind their members further using language or other means (this said, in many ant species the colonies are actually groups of families, and thus are genetically heterogeneous). Therefore, if the hypothesis advanced in this note is correct, their languages should be much simpler and less diverse. While their relative (with respect to humans') simplicity is perhaps a foregone conclusion, the diversity aspect appears an interesting subject for a comparative study.

\section{References}

Chomsky, N. (2015). Some core contested concepts. Journal of Psycholinguistic research, 44(1):91-104.

Corballis, M. C. (2017). Language evolution: a changing perspective. Trends in cognitive sciences, 21(4):229-236. 
Darwin, C. (1871). The descent of man: and selection in relation to sex. John Murray, Albemarle Street.

Hoshower, L. M., Buikstra, J. E., Goldstein, P. S., and Webster, A. D. (1995). Artificial cranial deformation at the omo m10 site: A tiwanaku complex from the moquegua valley, peru. Latin American Antiquity, 6(2):145-164.

Ludvico, L. R. and Kurland, J. A. (1995). Symbolic or not-so-symbolic wounds: The behavioral ecology of human scarification. Ethology and Sociobiology, 16(2):155-172.

Mackie, G. (1996). Ending footbinding and infibulation: A convention account. American sociological review, pages 999-1017.

Rand, D. G. and Nowak, M. A. (2013). Human cooperation. Trends in cognitive sciences, 17(8):413-425.

Reznikova, Z. (2007). Animal intelligence: From individual to social cognition. Cambridge University Press.

Reznikova, Z. and Ryabko, B. (2011). Numerical competence in animals, with an insight from ants. Behaviour, 148(4):405-434.

Tattersall, I. (2016). A tentative framework for the acquisition of language and modern human cognition. J. Anthropol. Sci, 94:157-166.

Traulsen, A. and Nowak, M. A. (2006). Evolution of cooperation by multilevel selection. Proceedings of the National Academy of Sciences, 103(29):10952-10955. 